\title{
Integrated Scheduling Optimization of Yard Crane and Yard Truck in Ship-loading Operation
}

\author{
Pengliang Cao ${ }^{1, a}$, Guiyan Jiang ${ }^{* 1,2,3, b}$, Hongran Zhao ${ }^{1, \mathrm{c}}$ \\ ${ }^{1}$ Faculty of Maritime and Transportation. Ningbo University \\ ${ }^{2}$ National Traffic Management Engineering \& Technology Research Centre Ningbo University \\ Sub-centre \\ ${ }^{3}$ Jiangsu Province Collaborative Innovation Center for Modern Urban Traffic Technologies) \\ adreamcpl@126.com, bjiangguiyan@nbu.edu.cn, czhaohongran0476@163.com
}

\begin{abstract}
Key words. Container terminal; Loading operation; Yard Crane; Yard Truck; Integrated scheduling optimization

Abstract. To further decrease turnaround time of container vessels, synchronous operation of Quay Cranes (QCs), Yard Cranes (YCs) and Yard Truck (YTs) is mainly considered in multiple work lines condition and an integrated scheduling optimization model of YC and YT, aiming at minimize the makespan of loading operation, is proposed with the consideration of practical constrains. According to complexity of the mathematical model, the multi-layers genetic algorithm (MLGA) is introduced to allocate the tasks of QCs and YCs and FCFS rule is used to dispatch YTs. Additionally, a YC dynamic scheduling strategy is proposed to further improve the solving efficiency of MLGA. In the end, effectiveness of the model and algorithm is certified by making numerical experiments.
\end{abstract}

\section{Introduction}

Quay Cranes (QCs), Yard Cranes (YCs) and Yard Truck (YTs) are three main handling equipment in container terminals. Generally, the existing academic works about the allocating and scheduling optimization of those equipment are prolific. However, most of them focus on single subproblem ${ }^{[1-5]}$, which ignored the integrity of handling system in container terminals. Along with the developing of optimization theory and technologies, the integrated scheduling of handling equipment is receiving increasing attention in academe and industry. However, after reviewing previous related research ${ }^{\text {[6-12] }}$, we find that the existing integrated YC and YT scheduling optimizing research all based on work line strategy, which is difficult to satisfy the need of increasingly busy production activities in container terminals. Facing the existed problem, this paper proposed an integrated YC and YT scheduling optimization model based on pool strategy to minimize the makespan of loading operation. MLGA and a simulation model are combined to solve the proposed problem. Plus, a dynamic dispatching rule for $\mathrm{YC}$ is proposed to further improve the solving efficiency.

\section{Formulation}

Problem description. To reasonably model the integrated $\mathrm{YC}$ and $\mathrm{YT}$ scheduling optimizing problem, this paper makes assumptions as follows.

- Only the loading operation is considered.

- The quantity and positions of outbound containers to be handled are given.

- Containers with the same properties are normally arranged in the same group. Therefore, we refer to the operation of such a group of containers as a task and each container as a job.

- The tasks that consists of enormous containers are normally stored in several blocks. Thus, YCs are not allowed to transfer blocks unless it finish the jobs belong to same task in a block. 
- The handling tasks in each work line should satisfy the task preference, but the tasks of different work lines have noninterference with each other. Each QC represents one work line.

- Each YT can only transport one container one time.

\section{Parameters.}

$t \quad$ index of operating time, $t=1,2, \ldots, T$.

$k \quad$ index of loading jobs, $k=1,2, \ldots, K$.

$r \quad$ index of work lines, $r=1,2, \ldots, R$.

$l \quad$ index of QCs, $l=1,2, \ldots, L$.

$m \quad$ index of YCs, $m=1,2, \ldots, M$.

$n \quad$ index of YTs, $n=1,2, \ldots, N$.

$U_{k}^{r} \quad$ index of task number of job $k$ in work line $r$.

$B_{m}^{t} \quad$ bay number of the location of YC $m$ at time $t$.

$W_{i, j}^{t} \quad$ number of YCs transfer from block $i$ to block $j$ at time $t, i, j \in R$.

$t_{l} \quad$ processing time of QC for each job.

$t_{m} \quad$ processing time of YC for each job.

$t_{n \text {.Rea }}^{l}$ reach time for $\mathrm{YT} n$ to $\mathrm{QC} l$.

$t_{m \text { Rea } a}^{k}$ reach time for $\mathrm{YC} m$ to bay of job $k$.

$t_{n \text {.Rea }}^{k}$ reach time for YT $n$ to bay of job $k$.

$\theta \quad$ a big positive number.

\section{Decision variables.}

$X_{k, t}^{l} \quad X_{k, t}^{l}=1$, if QC $l$ starts to operate job $k$ at time $t ; X_{k, t}^{l}=0$, otherwise.

$X_{k, t}^{m} \quad X_{k, t}^{m}=1$, if YC $m$ starts to operate job $k$ at time $t ; X_{k, t}^{m}=0$, otherwise.

$X_{k, t}^{n} \quad X_{k, t}^{n}=1$, if YT $n$ starts to operate job $k$ at time $t ; X_{k, t}^{n}=0$, otherwise.

$Y_{i, j}^{m} \quad Y_{i, j}^{m}=1$, if job $i$ is handled before job $j$ by YC $m ; Y_{i, j}^{m}=0$, otherwise.

$Y_{i, j}^{n} \quad Y_{i, j}^{n}=1$, if job $i$ is handled before job $j$ by YT $n ; Y_{i, j}^{n}=0$, otherwise.

\section{Mathematical model.}

$$
\min f=\max \left\{\sum_{t=1}^{T} t \cdot X_{k, t}^{l}+t_{l}\right\}
$$

Objective function (1) is to minimize the makespan of loading operation.

Subject to

$$
\begin{gathered}
\sum_{l=1}^{L} \sum_{t=1}^{T} X_{k, t}^{l}=\sum_{m=1}^{M} \sum_{t=1}^{T} X_{k, t}^{m}=\sum_{n=1}^{N} \sum_{t=1}^{T} X_{k, t}^{n}=1 \quad \forall k \\
\sum_{k=1}^{K} Y_{i, j}^{m}=\sum_{k=1}^{K} Y_{j, i}^{m}=1 \quad \forall i, j \in K, \forall m \\
\sum_{k=1}^{K} Y_{i, j}^{n}=\sum_{k=1}^{K} Y_{j, i}^{n}=1 \quad \forall i, j \in K, \forall n \\
\sum_{j=1}^{R} W_{i, j}^{t}+W_{i, i}^{t}-\sum_{j=1}^{R} W_{j, i}^{t} \leq 2 \quad \forall i, j \in M, \forall t \\
Y_{k, t}^{m} \cdot t \geq \max \left\{t_{m . \text { Rea }}^{k} t_{n . \text { Rea }}^{k}\right\} \quad \forall m, n, k \\
\left|B_{i}^{t}-B_{j}^{t}\right| \geq 1 \quad \forall i, j \in M, \forall t \\
X_{k, t}^{l} \cdot t \geq t_{n . \text { Rea }}^{l} \quad \forall n, l, k \\
X_{k, t}^{m} \leq \theta \cdot\left(Y_{k, t}^{m}-t_{m}\right) \quad \forall m, k, t
\end{gathered}
$$




$$
\begin{gathered}
X_{k, t}^{n} \leq \theta \cdot\left(Y_{k, t}^{n}-t_{m}\right) \quad \forall n, k, t \\
\max \left\{t_{m, \text { Rea }}^{i}, t_{n, \text { Rea }}^{i}\right\}-\max \left\{t_{m, \text { Rea }}^{j}, t_{n, \text { Rea }}^{j}\right\} \leq \theta \cdot\left(U_{j}^{r}-U_{i}^{r}\right) \quad \forall i, j \in K, \forall r
\end{gathered}
$$

\section{Algorithm}

It has been approved that the integrated scheduling problem of YC and YT is NP-hard ${ }^{[8]}$. Exact algorithms are not likely to get a feasible solution. Thus, a MLGA with two layers, namely main-layer and sub-layer, is introduced to solve the model, which are used to search task sequences for QCs and YCs respectively. Due to the computational intractability, a simulation model is developed for evaluating the solution presented by chromosomes. Additionally, a YC dynamic scheduling strategy is also introduced to further improve the solving efficiency of MLGA.

Structure of individuals. Individuals of the main-layer represent candidates of task sequences of QCs and those of the sub-layer are used to express the sequences of YCs to deal with those tasks. To show the generating process of two layers individuals, an example with 8 tasks, 2 QCs and 4YCs is represented, we assume the tasks assigned to QC1 and QC2 are $\{1,3,4,6\}$ and $\{2,5,7,8\}$.

Task Number
Storage Block

\begin{tabular}{|c|c|c|c|c|c|c|c|}
\hline 1 & 2 & 3 & 4 & 5 & 6 & 7 & 8 \\
\hline 3,4 & 5 & 7 & 6,8 & 3,5 & 4 & 6 & 7,8 \\
\hline
\end{tabular}

Fig. 1 The storage information of each task

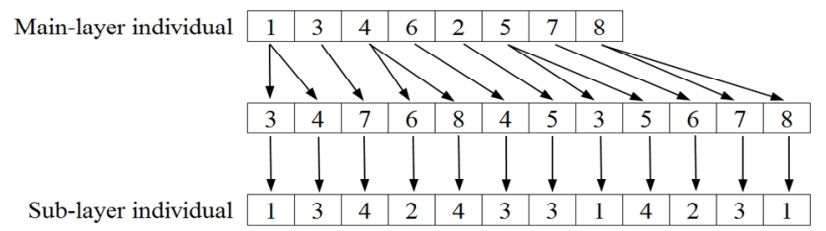

Fig. 2 An example of a solution representation

The storage information of each task is shown in Fig. 1 and

Fig. 2 represents the individual structure of each layer and the relationship of them. The main-layer individual represents a possible solution with 8 tasks served by 2 QCs are QC1: $1 \rightarrow 3 \rightarrow 4 \rightarrow 6$ and QC2: $2 \rightarrow 5 \rightarrow 7 \rightarrow 8$. In sub-layer individual, each gene is a $\mathrm{YC}$ dispatched to deal with the corresponding task stored in certain block.

Genetic operations. Generally, crossover operator is used for exploiting optimal solution, while mutation operator for expanding the search space.

a) Crossover operation. Integer crossing method is introduced in this paper. Since each task allocated to one QC cannot be replaced by the task allocated to another QC, crossover operation and mutation operation can only be operated in the fragment corresponding to certain QC to avoid the disorder of the tasks between QCs in main-layer individuals. By contrast, each gene in sub-layer individuals is independent to others, so there is no same constraints for sub-layer individuals.

b) Mutation operation. In this paper, a swap mutation operation is used for both layer of individuals, namely exchanging the positions of two genes in certain part of individuals to generate new offsprings. Besides, the operation objects in each layer have been mentioned in crossover part.

Dynamic scheduling. A simulation model is developed according to the real work process to compute the makespan of scheduling plan given by chromosomes and dispatch YTs with the FCFS rule. Additionally, considering the actual operation environment in container terminals have enormous equipment, this paper adopt time-driven simulation. Additionally, considering the individual length of sub-layer will obviously increase in large size conditions and can easily lead to the boosting in computing interval, a YC dynamic scheduling strategy is proposed to replace the iteration in sub-layer of MLGA. We extend the core idea of FCFS into YC dynamic scheduling by directly dispatching free YCs to serve the QCs that need to be served. Besides, we also take the job average distribution of YCs into consideration, which is beneficial to improve the average utilization ratio of YCs and the salary average level of its operators in container terminals. Thus, the 
YC with the fewest job amount will be dispatched in priority to serve QCs.

\section{Computational Experiments}

Experimental Settings. There are 3 QCs, 5 YCs and 10YTs in the computational experiments and the storage information about each task is shown in Table 1, including the storage block and the corresponding job amount in each block, besides, the tasks for each QC to tackle are also illustrated. Therefore, it is a large scale experiment scene with 472 jobs belonging to 10 tasks.

The processing time of a job by QCs and YCs are $30 \mathrm{~s}$ and $60 \mathrm{~s}$, YCs can travel a bay in $4 \mathrm{~s}$ and the speed of YTs is $5 \mathrm{~m} / \mathrm{s}$. Pure MLGA and the dynamic scheduling strategy are experimented in the same condition and these experiments are performed by Matlab 2012b. After test for several times with control variable method, we set the crossover and mutation coefficients as 0.7 and 0.1 respectively, in addition, the iteration of MLGA and dynamic scheduling strategy are limited into 200.

Table 1 The storage information about each task

\begin{tabular}{|c|c|c|c|c|c|c|c|c|}
\hline \multicolumn{3}{|c|}{ Tasks for QC1 } & \multicolumn{3}{|c|}{ Tasks for QC2 } & \multicolumn{3}{|c|}{ Tasks for QC3 } \\
\hline Task & Storage block & Job amout & Task & Storage block & Job amout & Task & Storage block & Job amout \\
\hline 1 & 1,5 & 32,24 & 5 & 4,6 & 32,36 & 8 & 2 & 48 \\
\hline 2 & 4 & 32 & 6 & 1,6 & 32,24 & 9 & 3 & 48 \\
\hline 3 & 3 & 40 & 7 & 4,5 & 28,36 & 10 & 3,6 & 16,16 \\
\hline 4 & 2 & 28 & & & & & & \\
\hline
\end{tabular}

\section{Computational Results}

Table 2 The task sequences of QCs and YCs

\begin{tabular}{cccccc}
\hline \multirow{2}{*}{ YC } & \multicolumn{2}{c}{ MLGA } & & \multicolumn{2}{c}{ Dynamic scheduling } \\
\cline { 2 - 3 } \cline { 5 - 6 } \cline { 5 - 6 } & Task sequence & Job sum & & Task sequence & Job sum \\
\hline 1 & $(5,4) \rightarrow(6,1) \rightarrow(8,2)$ & 112 & & $(5,4) \rightarrow(6,1) \rightarrow(7,4)$ & 92 \\
2 & $(4,2) \rightarrow(6,6) \rightarrow(1,5)$ & 76 & & $(4,2) \rightarrow(6,6) \rightarrow(1,5)$ & 76 \\
3 & $(5,6) \rightarrow(10,3) \rightarrow(7,4)$ & 80 & & $(5,6) \rightarrow(10,3) \rightarrow(8,2)$ & 100 \\
4 & $(9,3) \rightarrow(10,6) \rightarrow(7,5)$ & 100 & & $(9,3) \rightarrow(10,6) \rightarrow(7,5)$ & 100 \\
5 & $(2,4) \rightarrow(1,1) \rightarrow(3,3)$ & 104 & & $(2,4) \rightarrow(1,1) \rightarrow(3,3)$ & 104 \\
Max gap & & 36 & & & 28 \\
\hline
\end{tabular}

After computing, the same optimal solution of QCs is given by pure MLGA and the dynamic scheduling strategy, which is QC1: $4 \rightarrow 2 \rightarrow 1 \rightarrow 3$, QC2: $5 \rightarrow 6 \rightarrow 7$ and QC3: $9 \rightarrow 10 \rightarrow 8$. However, MLGA and dynamic scheduling strategy get different solutions in scheduling YCs as is shown in Table 2, in which $(5,4)$ represents the jobs that the task 5 stored in block 4 . It can also be seen that the job sum of all YCs are more average in the dynamic scheduling result.

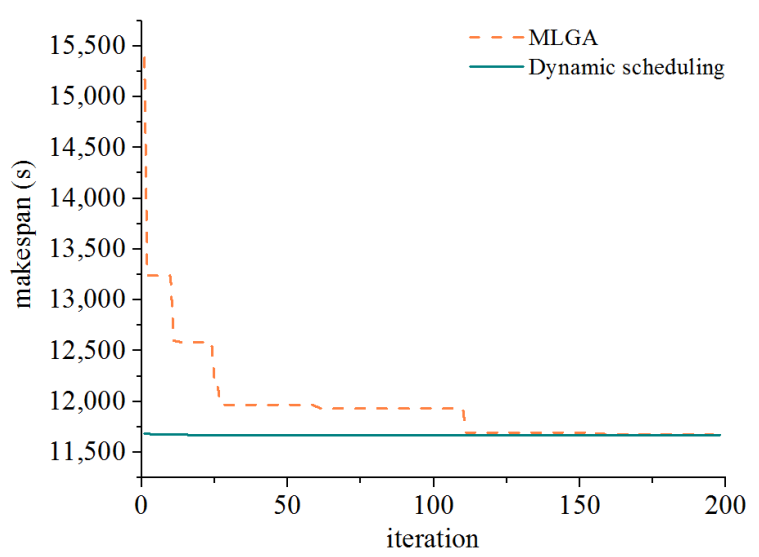

Fig. 3 The performance comparison in large size instance 
Fig. presents the convergence process of MLGA and the dynamic scheduling strategy. The former obtained the objective value 11672 by iterating 194 generations with $4052 \mathrm{~s}$, while after applying the dynamic scheduling strategy, the algorithm only need 324 s to achieve the same value result. It is obvious that the dynamic scheduling strategy improve the solving efficiency of MLGA.

\section{Conclusion}

The optimization model of the YC and YT integrated scheduling is established based on the YTs pool strategy. Through the large scale of the experiment, it confirms that the model of this paper can provide optimization solutions for integrity and coordination of operations in container terminals.

In solving algorithm aspect, considering the model complexity, a YC dynamic scheduling strategy is introduced to replace the iteration process of sub-layer in MLGA, and successfully improved the computational efficiency and reduce the max gap in job amount among YCs, which can not only improve the average utilization ratio of YCs but also the salary average level of YC operators in container terminals. Through the application of this improvement measure, the solving algorithm can perform more steadily and effectively.

\section{Acknowledgement}

This work was financially supported by Pao Yu-kong and Pao Zhao-long Scholarship For Chinese Students Studying Abroad, 2017 Zhejiang College students' scientific and technological innovation activities program.

\section{References}

[1] Shao Q Q, XuQ, Bian Zh, et al. Stockpiling operating optimization for yard crane with containers delivery time uncertainty[J]. Systems Engineering - Theory \& Practice, 2015, 35(02):394-405.

[2] Li W, Wu Y, Petering M E H, et al. Discrete time model and algorithms for container yard crane scheduling[J]. European Journal of Operational Research, 2009, 198(1):165-172.

[3] Liang C J, Chen M, Gen M, et al. A multi-objective genetic algorithm for yard crane scheduling problem with multiple work lines[J]. Journal of Intelligent Manufacturing, 2014, 25(5):1013-1024.

[4] Ding R T. Dispatch optimization strategy of port container trucks based on collaborative capacity constraints[J]. Journal of Tsinghua University (Science and Technology), 2012(8):1158-1164.

[5] Tao J, Qiu Y. A simulation optimization method for vehicles dispatching among multiple container terminals[J]. Expert Systems with Applications, 2015, 42(7):3742-3750.

[6] Chen L, Bostel N, Dejax P, et al. A tabu search algorithm for the integrated scheduling problem of container handling systems in a maritime terminal[J]. European Journal of Operational Research, 2007, 181(1):40-58.

[7] Cao J, Shi Q, Lee D H. Integrated Quay Crane and Yard Truck Schedule Problem in Container Terminals[J]. Tsinghua Science and Technology, 2010, 15(4):467-474.

[8] Cao J X, Lee D H, Chen J H, et al. The integrated yard truck and yard crane scheduling problem: Benders' decomposition-based methods[J]. Transportation Research Part E Logistics \& Transportation Review, 2010, 46(3):344-353.

[9] Le M, Toshitake, Kohmura. An Improved Model for the Integrated Yard Crane and Yard Truck Scheduling Problem[J]. Josai Journal of Business Administration, 2013, 10(3):29-41.

[10] Chang W A, Ramakrishna R S. A genetic algorithm for shortest path routing problem and the 
sizing of populations[J]. IEEE Transactions on Evolutionary Computation, 2003, 6(6):566-579.

[11] Hwang S F, He R S. A hybrid real-parameter genetic algorithm for function optimization[J]. Advanced Engineering Informatics, 2006, 20(1):7-21.

[12] Oreski S, Oreski G. Genetic algorithm-based heuristic for feature selection in credit risk assessment[J]. Expert Systems with Applications, 2014, 41(4):2052-2064. 